\title{
EXPLORANDO O POTENCIAL DOS JOGOS DIGITAIS: UMA REVISÃO SOBRE A UTILIZAÇÃO DOS EXERGAMES NA EDUCAÇÃO
}

\author{
EXPLORANDO EL POTENCIAL DE LOS JUEGOS DIGITALES: UNA \\ REVISIÓN SOBRE EL USO DE EXERGAMES EN LA EDUCACIÓN
}

\author{
EXPLORING THE POTENTIAL OF DIGITAL GAMES: A REVIEW ON THE USE \\ OF EXERGAMES IN EDUCATION
}

Adilson Rocha FERREIRA ${ }^{1}$

Deise Juliana FRANCISCO ${ }^{2}$

RESUMO: O objetivo deste trabalho é mapear a produção sobre a utilização dos exergames na educação dentre os anos de 2010 e 2015. Inicialmente, foram realizadas buscas no Periódicos CAPES/MEC e na Biblioteca Virtual em Saúde. Contudo, não obtivemos êxito com as buscas, onde não foram encontrados trabalhos na área. Após isso, foi feita uma busca no Periódicos Qualis da Plataforma Sucupira por periódicos que tratam especificamente da temática dos jogos digitais e suas relações com diversas áreas de conhecimento. Com isso, realizamos uma "garimpagem", durante os meses de julho e agosto de 2015, nos seguintes periódicos online: International Journal of Computer Games Technology, G|A|M|E - Games as Arts, Media, Entertainment e Games for Health Journal. Foram analisados 5 trabalhos primários e os resultados destas pesquisas indicam que a implementação e a prática dos exergames possibilita o aumento do nível de atividade física, chegando a níveis moderados e vigorosos, sendo benéfica à saúde, tanto do ponto de vista físico quanto cognitivo. Entretanto, há a necessidade de que mais investigações sejam realizadas, envolvendo diferentes desenhos experimentais com maior duração das intervenções e com a participação de pessoas de diferentes níveis de ensino, a fim de consolidar os achados, pois há poucos estudos e os resultados ainda são preliminares.

PALAVRAS-CHAVE: Videogame. Kinect. Ensino e aprendizagem.

RESUMEN: El objetivo de este estudio es el mapeo de la literatura sobre el uso de exergames en la educación de los años 2010 y 2015. Inicialmente, se realizaron búsquedas en las publicaciones periódicas CAPES / MEC y la Biblioteca Virtual en Salud. Sin embargo, no habíamos busca éxito donde no se encontraron estudios en el área. Después de eso, se hizo una búsqueda en la plataforma Qualis Periódicos Sucupira para las revistas que tratan específicamente de la cuestión de los juegos digitales y sus relaciones con las diversas áreas del conocimiento. Por lo tanto, se realizó un "minero" durante los meses de julio y agosto de 2015, las siguientes revistas

\footnotetext{
${ }^{1}$ Universidade Federal de Alagoas (UFAL), Alagoas - Brasil. Especialista em Mídias na Educação pelaBrasil). Mestrando do Programa de Pós-graduação em Educação. E-mail: adilsonrf.al@ gmail.com

${ }^{2}$ Universidade Federal de Alagoas (UFAL), Alagoas - Brasil. Professora do Programa de Pós-graduação em Educação. Doutora em Informática na Educação pela Universidade Federal do Rio Grande do Sul (UFRGS - Brasil). E-mail: deisej@gmail.com
} 
en línea: Revista Internacional de Juegos de tecnología de la computación, $G|A| M \mid$ E - Juegos de las artes, los medios de comunicación, entretenimiento y juegos para la Salud Diario. Se analizaron 5 de trabajo principal y los resultados de esta investigación indican que la aplicación y la práctica de exergames permite que el mayor nivel de actividad física, llegando a niveles moderados a vigorosos que son beneficiosos para la salud, tanto física como punto de vista cognitivo. Sin embargo, existe la necesidad de una mayor investigación, la participación de diferentes diseños experimentales con una mayor duración de las intervenciones y la participación de personas de diferentes niveles de la educación con el fin de consolidar los resultados, ya que hay pocos estudios y los resultados son todavía preliminares.

PALAVRAS CLAVE: Video juego. Kinect. La enseñanza y el aprendizaje.

ABSTRACT: The objective of this work is to map the academic production on the use of exergames in education between the years of 2010 and 2015. Initially, searches were done in the CAPES/MEC Periodicals and in the Virtual Health Library. However, we did not succeed with the searches, where no papers were found in the area. After that, a search was made in the Periodicals Qualis of the Sucupira Platform by periodicals that deal specifically with the theme of the digital games and their relations with several areas of knowledge. With this, we performed a "garimpagem", during the months of July and August of 2015, in the following online newspapers: International Journal of Computer Games Technology, $G|A| M \mid E$ - Games as Arts, Media, Entertainment and Games for Health Journal. Five primary studies were analyzed and the results of these studies indicate that the implementation and the practice of exergames allows the increase of the level of physical activity, reaching moderate and vigorous levels, being beneficial to the health, both from the physical and cognitive point of view. However, is needed more research, involving different experimental designs with longer duration of interventions and with the participation of people of different education levels, in order to consolidate the findings, since there are few studies and the results are still preliminary.

KEYWORDS: Videogame. Kinect. Teaching and learning.

\section{Introdução}

Devido ao grande avanço tecnológico, particularmente no que concerne aos dispositivos eletrônicos, as Tecnologias Digitais da Informação e Comunicação (TDIC) estão cada vez mais presentes no âmbito educacional, impulsionando transformações significativas, o que proporciona diferentes formas de ensino e aprendizagem (FROSI; SCHLEMMER, 2010). Isso indica que a sociedade vem se apropriando das funcionalidades das TDIC, de modo a incorporá-las em diversas funções, dentre as quais podemos destacar a contribuição no âmbito educacional. Desse modo, o 'fazer', e até mesmo o 'pensar', são constantemente reconfigurados diante da imensidão de 
possibilidades oferecidas pelas mídias digitais (BARACHO; GRIPP; LIMA, 2012). Essa prática de convergência presente em nossas relações é o que Lévy (1999) e Lemos (2002) denominam de cibercultura.

Inicialmente, os videogames foram desenvolvidos com fins apenas lúdicos, com foco no entretenimento, onde o objetivo era proporcionar uma forma diferenciada de lazer. Ao passar do tempo, estudiosos identificaram potencialidades nos jogos digitais que poderiam ser exploradas em diversos âmbitos, sendo um deles a educação, deixando o aspecto do entretenimento para ocupar lugar de destaque em âmbito educacional (BARBOZA; SILVA, 2014).

Por muito tempo, a atividade de jogar videogames foi encarada como um hábito não-saudável, propiciando o aparecimento de doenças como hipertensão, diabetes, dentre outras (PAPASTERGIOU, 2009). Contudo, com a evolução do mercado de games, essa visão vem sendo alterada, pois já é possível controlar os videogames utilizando os movimentos do corpo humano como método de entrada, extrapolando o simples ato de apertar botões.

Os exergames, classe de jogos que utiliza o movimento humano para interagir com o game, o que antes era possível apenas com controles manuais, teve uma maior popularização a partir da sétima geração de games, com a aparição dos consoles ${ }^{3}$ caseiros Playstation 3 (Sony Computer Entertainment), Nintendo Wii (Nintendo) e Kinect para Xbox 360 (Microsoft), proporcionando uma melhor experiência de imersão, além de aumentar o nível de atividade física do jogador $(\mathrm{OH}$; YANG, 2010). Os exergames vêm ganhando espaço entre investigadores de diversas áreas do conhecimento, sendo a educação uma delas, uma vez que a utilização dessa classe de games tem mostrado resultados positivos ao aumentar o nível de atividade física e, consequentemente, diminuir o comportamento sedentário dos jogadores (MELLECKER; MCMANUS, 2013).

Tendo em vista a convergência das mídias digitais e as potencialidades pedagógicas dos games, em específico os exergames, surgiu a curiosidade em investigar como os exergames estão sendo estudados em âmbito escolar. Com isso, o problema da pesquisa foi o seguinte: de que forma os autores estão tratando em suas produções a

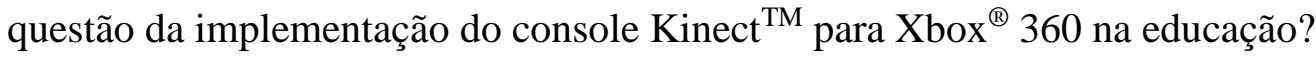

\footnotetext{
${ }^{3}$ Microcomputador dedicado a executar jogos de vídeo (videogames) de diversos gêneros, como jogos de ação, jogos de esportes, jogos de aventuras, dentre outros. Os videogames recebem a denominação de console pelo fato de utilizar outro aparelho, no caso a TV, para seu funcionamento.
} 
Dentre os consoles da sétima geração de games, optou-se por investigar a produção acerca da implementação do Kinect para Xbox 360 (Microsoft) em âmbito educacional devido ao fato deste console simular com mais fidelidade a realidade, uma vez que não é preciso segurar nenhum implemento para reconhecimento do movimento, como é imprescindível para que ocorra a interação no Playstation 3 (Sony Computer Entertainment) e Nintendo Wii (Nintendo).

Diante desse cenário, este trabalho teve como objetivo geral mapear a produção sobre a utilização dos exergames na educação. Como objetivos específicos, teve-se: apresentar uma síntese dos trabalhos empíricos que incorporaram este segmento de games como uma ferramenta potencializadora de aprendizagem, identificar a faixa etária dos sujeitos, nível de ensino em que os exergames foram aplicados e os games utilizados, e, por fim, analisar as possíveis implicações na cognição e bem-estar das pessoas que utilizaram os exergames.

Este trabalho foi estruturado em 5 seções. A primeira contextualiza o trabalho; a segunda trata os games como instrumento pedagógico potencializador da aprendizagem; a terceira seção aborda o percurso metodológico adotado para nortear $\mathrm{o}$ desenvolvimento deste estudo; na quarta seção os resultados obtidos com a revisão sistemática de literatura são expostos e discutidos e na quinta seção são apresentadas as considerações finais e perspectivas para futuros estudos sobre a temática.

\section{Os games como instrumento pedagógico potencializador da aprendizagem}

Sendo uma das mais antigas atividades da humanidade, os jogos são considerados como um expoente cultural da sociedade como um todo (GULARTE, 2010). Segundo Huizinga (2014), o jogo é elemento constituinte da cultura humana, uma vez que este é anterior à cultura. Ainda de acordo com o autor, o jogo pode ser definido como:

[...] uma atividade ou ocupação voluntária, exercida dentro de certos e determinados limites de tempo e espaço, segundo regras livremente consentidas, mas absolutamente obrigatórias, dotado de um fim em si mesmo, acompanhado de um sentimento de tensão e de alegria e de uma consciência de ser diferente da "vida cotidiana" (HUIZINGA, 2014, p. 33). 
Com essa abordagem, busca-se, a partir do jogo, a possibilidade de transformação da realidade em que o jogador se encontra, fugindo da confusão da vida e da imperfeição do mundo para uma perfeição temporária e limitada, levando-o a uma reflexão sobre a prática do jogo (HUIZINGA, 2014). Quando o jogador está em contato com o jogo, ele cria para si um pequeno mundo paralelo ao real, regido pelas regras do jogo e pela sua cultura (MOITA, 2007).

Por jogos eletrônicos, ou videogames, consideram-se aqueles "aparelhos que que interagem com seus usuários por meio de interfaces homem-máquina, gerando respostas sensoriais" (GULARTE, 2010, p. 39). Ao longo do tempo, várias tentativas de atingir essas interações, com e sem sucesso, foram feitas e algumas delas continuam no cotidiano de muitos lares, onde os jogos eletrônicos estão cada vez mais acessíveis e mais populares.

Diante dessa visível popularização, os jogos digitais estão sendo empregados em diversos setores além do entretenimento. Antes com fins apenas lúdicos, agora os jogos assumem variados papeis, onde podemos ressaltar o viés educacional, campo de investigação relativamente recente, mas que está em crescente interesse por parte de pesquisadores da área da educação (BARBOZA; SILVA, 2014).

A utilização de games na educação não possibilita apenas a modernização do ensino e da aprendizagem, como também atrai os envolvidos para a participação na construção do conhecimento, sendo elemento ativo nesse processo.

Por se tratar de uma tecnologia relativamente recente, a produção sobre a utilização dos games como ferramenta pedagógica e suas implicações na educação é escassa, mas em crescente interesse, tendo em vista a potencialidade pedagógica desse objeto técnico.

Contudo, não há um consenso acerca da utilização dos jogos digitais. Por um lado, pesquisadores afirmam que os games podem ocasionar diversos tipos de problemas, como por exemplo lesões, introversão social, estimular comportamento agressivo (PAPASTERGIOU, 2009), como também associam esta prática ao sedentarismo e obesidade, estimulando um estilo de vida, portanto, nocivo à saúde (SOTHERN, 2004). Entretanto, por outro lado, com a evolução da experiência de imersão com a utilização do movimento para interagir com o game, há pesquisadores que defendem a utilização de videogames, por considerar que determinados aspectos educacionais podem ser trabalhados a partir da vivência nessa interface (ALVES, 2005; GEE, 2007; 2014; MOITA, 2007). 
Como todas as outras tecnologias, os jogos digitais por si só não determinam a viabilidade da prática ou não, pois isso depende de como eles serão utilizados para atingir os objetivos estabelecidos no planejamento prévio (GEE, 2007; 2014). Nos jogos eletrônicos, geralmente o objetivo é vencer. Contudo, investigadores vêm percebendo que os jogadores de videogames estão menos preocupados em atingir os objetivos do game e mais focados na diversão proporcionada pela experiência da imersão, o que pode potencializar a aprendizagem (SHAFER; CARBONARA, 2015).

Da mesma forma que a área de investigação sobre games e suas contribuições está em constante evolução, podemos notar que há também uma evolução no âmbito de desenvolvimento de videogames, proporcionado diferenciadas vivências, cada vez mais realistas. Ao logo dos últimos 50 anos, desde a sua criação até os dias atuais, os games evoluíram significativamente, passando de equipamentos de baixa qualidade gráfica até recursos tecnológicos de virtualidade em 3 dimensões, aumentando assim a experiência de imersão (FERES NETO, 2007).

Em 2010, a empresa Prime Sense desenvolveu e disponibilizou no mercado um dispositivo chamado Kinect $^{\mathrm{TM}}$ (ver Figura 1) para ser utilizado com o console Xbox 360 da empresa Microsoft, o que modificou a forma de interação com o usuário com o jogo. Sua finalidade é servir com ponto de ligação ao game, imergir à realidade virtual através do reconhecimento dos movimentos dos corpos dos jogadores e da transmissão desses movimentos ao jogo, suprimindo assim o uso dos joysticks ${ }^{4}$ (CORRÊA et al, 2011). De acordo com Paula (2011), o Kinect ${ }^{\mathrm{TM}}$ possui duas câmeras, um sensor de profundidade, um microfone embutido e um motor que controla o seu ângulo de inclinação.

Figura 1: Estrutura física do Kinect para Xbox 360.

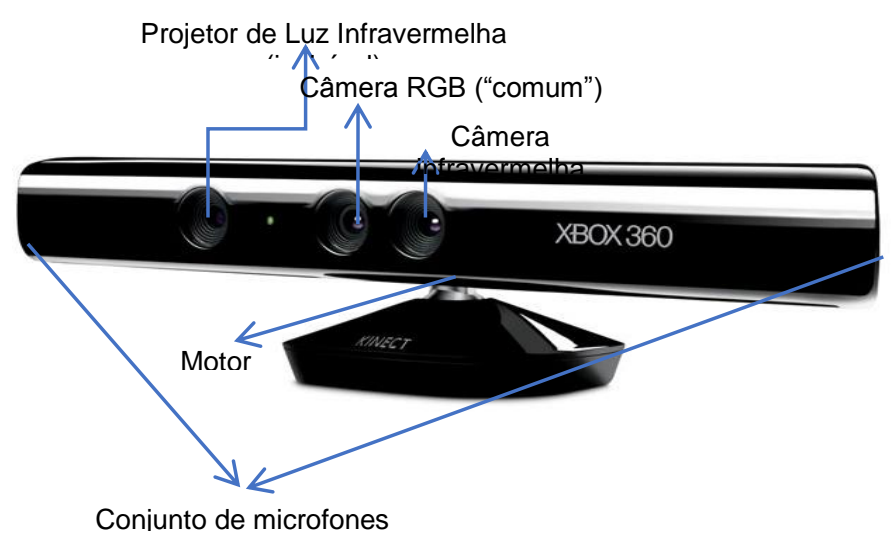

Fonte: Paula (2011).

\footnotetext{
${ }^{4}$ Periférico utilizado em jogos de computador ou videogames, dotado de um ou mais botões capazes de comandar certas ações no jogo ao serem pressionados.
} 
Além de apertar botões, agora é possível interagir com os games utilizando os movimentos do próprio corpo como método de entrada de comandos, dando origem a uma classe de games. Ainda não há um consenso sobre sua nomenclatura, mas pode ser denominada por exergames (EXG), o que reflete a junção do exercício físico à atividade de jogar videogame (VAGHETHI; BOTELHO, 2010).

O Kinect ${ }^{\mathrm{TM}}$ para $\mathrm{Xbox}^{\circledR} 360$ se destaca dos demais da categoria - Nintendo Wii e Playstation 3 - por não necessitar de nenhum outro periférico para interação com o game, como Wii Remote ou Kit Move, respectivamente. Com isso, o Kinect ${ }^{\mathrm{TM}}$ para Xbox $^{\circledR} 360$ é considerado econômico e seguro, pois não demanda gastos extras e diminui a possibilidade de acidentes, uma vez que são utilizados os movimentos naturais do corpo humano mapeados por um conjunto de sensores, sem a necessidade de estar segurando algum implemento.

Para a disciplina Educação Física, as características da geração de jogos com movimentos contrapõe a ideia de sedentarismo, nocividade à saúde, passividade e inatividade do jogador, pois é necessário mover-se para jogar, fornece novos desafios, o que requer uma discussão mais aprofundada sobre sua utilização como ferramenta didático-pedagógica.

\section{Percurso metodológico}

De acordo com os propósitos deste estudo, foi feita uma revisão sistemática de literatura sobre trabalhos que investigaram a utilização dos exergames em âmbito educacional. Inicialmente, foram realizadas buscas no Periódicos CAPES/MEC e na Biblioteca Virtual em Saúde utilizando as seguintes palavras-chave: "exergame"; "learning"; "teaching"; "Kinect". Contudo, não obtivemos êxito com as buscas, onde não foram encontrados trabalhos na área. Acreditamos que isto possa ter ocorrido por se tratar de um tema em crescente interesse, porém ainda pouco explorado.

Após isso, foi feita uma busca no Periódicos Qualis da Plataforma Sucupira ${ }^{5}$ por periódicos que tratam especificamente da temática dos jogos digitais e suas relações com diversas áreas de conhecimento. Com isso, realizamos uma "garimpagem", durante

\footnotetext{
${ }^{5}$ Ferramenta desenvolvida pela Comissão de Aperfeiçoamento de Pessoal do Nível Superior (CAPES) que tem como objetivos coletar informações, realizar análises e avaliações e ser a base de referência do Sistema Nacional de Pós-Graduação (SNPG).
} 
os meses de julho e agosto de 2015, nos seguintes periódicos online: International Journal of Computer Games Technology, G|A|M|E - Games as Arts, Media, Entertainment e Games for Health Journal.

Realizamos a busca de trabalhos publicados nos periódicos acima citados, com as mesmas palavras-chave. A busca foi limitada em artigos publicados entre os anos de

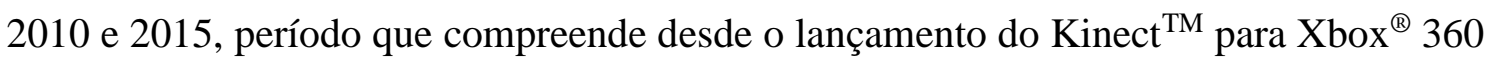
até a data da realização do presente estudo.

Uma vez realizada a busca, foi feita a leitura do título e do resumo dos trabalhos listados. Após isso, foram selecionados e transferidos para o computador os estudos que se relacionavam com a temática desse estudo para leitura completa e posterior análise. Foram selecionados para análise trabalhos empíricos que utilizaram os exergames em âmbito educacional, desde o nível básico ao superior, detalhados abaixo no Quadro 1.

Quadro 1: Descrição dos trabalhos selecionados.

\begin{tabular}{|c|c|c|c|c|}
\hline $\begin{array}{c}\text { Autor }(e s) ~ / ~ \\
\text { Ano }\end{array}$ & Sujeitos estudados & $\begin{array}{l}\text { Tipo do } \\
\text { estudo }\end{array}$ & Duração & Games utilizados \\
\hline $\begin{array}{l}\text { Clevenger e } \\
\text { Howe }(2015)\end{array}$ & $\begin{array}{c}58 \text { crianças e } \\
\text { adolescentes com } \\
\text { idade entre } 8 \text { e } 17 \\
\text { anos }\end{array}$ & Comparativo & $\begin{array}{c}2 \text { sessões }(\mathrm{EXG}) \\
\text { por dupla de } \\
\text { jogadores }\end{array}$ & 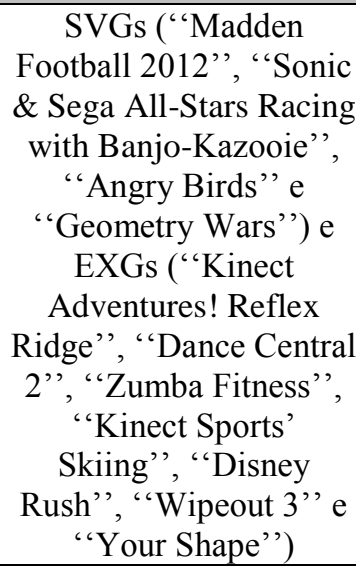 \\
\hline Lin (2015) & $\begin{array}{c}129 \text { universitários } \\
\text { com idade entre } 18 \text { e } \\
26 \text { anos }\end{array}$ & Experimental & $\begin{array}{l}1 \text { sessão (12 } \\
\text { minutos) de EXG }\end{array}$ & "'Just Dance 3"' \\
\hline $\begin{array}{l}\text { MacArthur et } \\
\text { al (2014) }\end{array}$ & $\begin{array}{c}16 \text { crianças com } \\
\text { idade entre } 5 \text { e } 8 \\
\text { anos }\end{array}$ & Comparativo & $\begin{array}{c}2 \text { sessões (15 } \\
\text { minutos) de EXG e } \\
\text { OP }\end{array}$ & $\begin{array}{l}\text { "Kinect Adventures! } \\
\text { River Rush", }\end{array}$ \\
\hline $\begin{array}{c}\text { Reading e } \\
\text { Prickett (2013) }\end{array}$ & $\begin{array}{c}41 \text { crianças com } \\
\text { idade entre } 5 \text { e } 12 \\
\text { anos }\end{array}$ & Experimental & $\begin{array}{c}1 \text { sessão de } \\
\text { familiarização (5 a } \\
10 \text { min })+1 \text { sessão } \\
\text { de } 20 \text { minutos de } \\
\text { EXG }\end{array}$ & "Kinect Adventures!" \\
\hline $\begin{array}{l}\text { Rosenberg } \\
\quad(2013)\end{array}$ & $\begin{array}{c}47 \text { crianças com } \\
\text { idade entre } 10 \text { e } 15 \\
\text { anos }\end{array}$ & Experimental & $\begin{array}{c}5 \text { sessões de } 1 \text { hora } \\
\text { (1 familiarização + } \\
4 \text { EXG) }\end{array}$ & $\begin{array}{l}\text { "Motion Sports } \\
\text { Adrenaline", "Sonic } \\
\text { Free Riders", "Virtua } \\
\text { Tennis 4", "Kinect } \\
\text { Sports", "Kinect } \\
\text { Adventures" e "Just } \\
\text { Dance 3"” } \\
\end{array}$ \\
\hline
\end{tabular}

Fonte: Autoria própria. 


\section{Resultados e discussão}

Como resultado da "garimpagem" de artigos nos periódicos International Journal of Computer Games Technology, G|A|M|E? Games as Arts, Media, Entertainment, International Journal of Computer Games Technology e Games for Health Journal, foram selecionados 5 estudos primários com base no título, resumo e conclusão dos artigos. Com relação à frequência de atividades de pesquisa, pode-se ver no Gráfico 1 a quantidade de artigo selecionados publicados nos periódicos acima citados entre os anos de 2010 e 2015.

Gráfico 1: Quantidade de artigos selecionados publicados por ano (2010-2015).

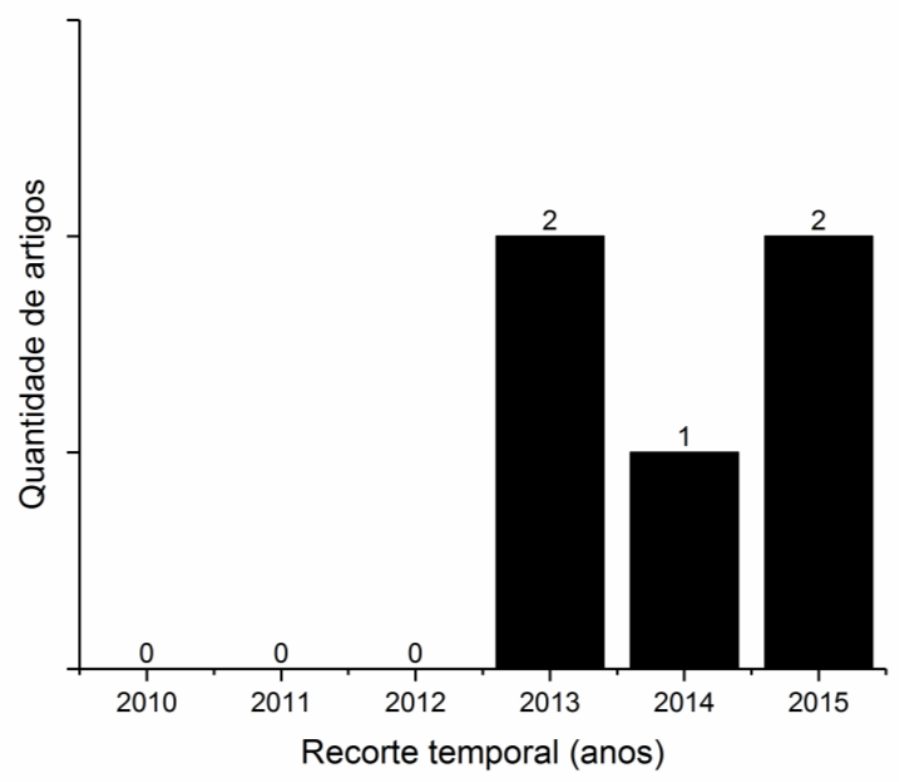

Fonte: Autoria própria.

Dentre os anos de 2010 a 2012, não foi desenvolvido nenhum estudo na área. Entretanto, a partir de ano de 2013, iniciaram-se as atividades de pesquisa e mantém-se uma certa regularidade das mesmas nos anos seguintes. Analisando o Gráfico 1, com relação à frequência e à quantidade de estudos primários desenvolvidos, é possível ver que a temática é recente e ainda pouco explorada no meio acadêmico, mas em crescente aumento de interesse por parte de investigadores. Isto pode decorrer do reconhecimento das potencialidades dos exergames para a aprendizagem e o bem-estar dos sujeitos, proporcionados pela prática lúdica e interativa de atividade física (VAGHETTI; BOTELHO, 2010). 
Clevenger e Howe (2015), investigadores da Faculdade de Ciências Aplicadas à Saúde e Bem-Estar da Universidade de Ohio, desenvolveram um estudo que teve como objetivo comparar o gasto energético de atividade física, intensidade e satisfação dos exergames (EXGs) com jogos de videogames jogados sentados (SVGs).

Crianças e adolescentes voluntárias, em um total de 58, com idade entre 8 e 17 anos, jogaram uma seleção aleatória de seis videogames ( 2 jogos de videogame jogados sentados seguidos de 4 exergames). Foram utilizados os seguintes jogos: ("Madden Football 2012”, "Sonic \& Sega All-Stars Racing with Banjo-Kazooie", "Angry Birds" e "Geometry Wars") e EXGs ("Kinect Adventures! Reflex Ridge", "Dance Central 2", “Zumba Fitness", “Kinect Sports' Skiing”, "Disney Rush”, "Wipeout 3" e "Your Shape"). Como protocolo, os sujeitos foram encaminhados para uma sala para jogar seis jogos (2 SVGs e 4 EXGs), com duração de 6 a 10 minutos cada e com intervalo de 4 minutos entre os jogos. Enquanto os participantes estavam jogando, foi mensurado o consumo total de oxigênio dos participantes e o nível de atividade física do jogo. Imediatamente após a cada jogo, no intervalo, foi feita uma série de questionamentos aos participantes com relação à percepção de satisfação do jogo, selecionando um rosto em uma escala de afetividade fácil com três itens.

Como resultados, Clevenger e Howe (2015) encontraram que a maioria dos EXGs foram classificados como uma atividade física de intensidade moderada a vigorosa, enquanto os SVGs foram classificados como baixa intensidade. Com relação à satisfação, não foram encontradas diferenças significativas entre EXGs e SVGs. Dessa forma, os autores concluíram que os AVGs exigem um gasto de energia suficiente para ser uma alternativa adequada ao jogo tradicional, contribuindo com os índices recomendados de atividade física segundo a World Health Organization (2010).

Lin (2015), pesquisadora do Departamento de Comunicação e Tecnologia da Universidade Nacional Chiao Tung, realizou um estudo que teve por objetivo examinar os efeitos da interatividade e uso de controles sobre as respostas fisiológicas e psicológicas dos participantes durante a prática do exergame.

Participaram desse estudo 129 estudantes universitários com idade entre 18 e 26 anos. Foi selecionado o jogo "Just Dance 3" para interação com os participantes, com e sem feedback (videogame e vídeo gravado do jogo de videogame sem feedback) e com e sem controle (PlayStation 3 e Xbox 360, respectivamente). Foram analisadas a frequência cardíaca e pressão arterial, bem como a percepção subjetiva de esforço e a vitalidade subjetiva. Como desenho do estudo, os participantes dançaram uma música 
como ensaio e aquecimento, seguidas de três músicas para o estudo, o que durou aproximadamente 12 minutos.

Não foram encontradas variâncias significativas dos resultados de frequência cardíaca, pressão arterial, movimentos corporais ou resultados psicológicos percebidos nas questões de interatividade e manipulação de controle. Dessa forma, Lin (2015) sugere que vídeos gravados de videogame também são ferramentas efetivas para alcançar níveis moderados de atividade física, o que pode suprir o acesso limitado aos exergames.

MacArthur et al (2014), da Universidade de Tennessee, realizaram uma pesquisa com o objetivo de comparar o percentual de tempo gasto em nível de atividade física moderado a vigoroso enquanto jogando exergames e atividades não-estruturadas ao ar livre.

Crianças com idade entre 5 e 8 anos, em um total de 16, participaram do estudo, onde completaram duas sessões de duração de 15 minutos de exergames e atividades não-estruturadas ao ar livre. Para a sessão de exergame, foi utilizado o jogo "Kinect Adventures! River Rush" e a sessão de atividades ao ar livre foi conduzida em um parque externo. Questionários demográficos foram realizados com os responsáveis das crianças. Dados antropométricos e alimentares das crianças foram registrados antes de cada sessão. Durante as intervenções, foram utilizados acelerômetros e a observação direta para mensurar o nível de atividade física das crianças enquanto jogando exergames e realizando atividades não-estruturadas no parque externo.

Os dados coletados demonstraram que houve maior percentagem de tempo gasto em nível moderado a vigoroso de atividade física enquanto jogando exergames em comparação com realização de atividades no parque ao livre. Nesse sentido, os resultados sugerem que os exergames podem se constituir de uma fonte para alcançar o nível moderado a vigoroso de atividade física em crianças.

Reading e Prickett (2013), pesquisadores da Faculdade de Cinesiologia da Universidade de New Brunswick, Canadá, desenvolveram uma pesquisa com o objetivo de determinar se os movimentos do corpo humano necessários para jogar o exergame provocaram uma atividade física de nível de intensidade moderado.

Fizeram parte desse estudo 41 crianças com idade entre 5 e 12 anos, sendo 26 meninos e 15 meninas que não possuem ou têm acesso regular aos exergames. Foi mensurado o nível de atividade física dos sujeitos enquanto jogando o exergame “Kinect Adventures!”. Antes dos dados experimentais serem coletados, os participantes 
realizaram uma sessão de familiarização de 5 a 10 minutos. Após a familiarização, os participantes descansaram e somente após isso puderam escolher as atividades do jogo “Kinect Adventures!" para jogar por 20 minutos. Imediatamente após a intervenção, os participantes responderam um questionário a respeito da satisfação dos exergames em comparação a outros tipos de jogos que eles já jogaram.

Com os resultados, verificou-se que as crianças ao jogar os exergames atingiram níveis moderados de intensidade de atividade física. Dessa forma, segundo Reading e Prickett (2013), os exergames, orientados para o uso adequado, podem reduzir o tempo sedentário e aumentar os níveis de atividade física moderados diários para crianças.

Rosenberg (2013), pesquisador da Faculdade de Ciências do Desporto, Exercício e Saúde da Universidade de Western, Austrália, investigou os exergames com o objetivo de medir o gasto de energia necessário para jogar sessões de 15 minutos de 6 jogos do console Kinect para Xbox 360 em sequência.

Participaram dessa pesquisa 47 crianças com idade entre 10 e 15 anos, sendo 19 meninas e 28 meninos. Para a realização das sessões de exergames - uma de familiarização e quatro experimentais, com duração de uma hora - foram utilizados os jogos "Motion Sports Adrenaline”, "Sonic Free Riders", "Virtua Tennis 4", "Kinect Sports", "Kinect Adventures" e "Just Dance 3", divididos em dois blocos de três jogos, onde 24 participantes jogaram o primeiro bloco e outros 23 jogaram o segundo. Antes de casa sessão, foi mensurada a frequência cardíaca de repouso, e após isso os participantes foram conduzidos a três sessões consecutivas de diferentes exergames, com intervalo de 2 minutos entre as sessões. Peso, altura, frequência cardíaca e gasto energético foram mensurados durante as sessões.

A média do gasto energético dos seis exergames foi significativamente maior do que o gasto energético em repouso, e pequenas diferenças foram encontradas com relação ao gasto energético necessário em cada jogo. Dessa forma, os jogos do Kinect para Xbox 360 utilizados nesse estudo foram classificados com um nível de intensidade de atividade física moderado ao longo das sessões. Apesar de pequenas diferenças no gasto energético terem sido encontradas entre os 6 jogos utilizados, todos eles são suscetíveis de contribuir para a diminuição do sedentarismo em crianças.

\section{Considerações finais}


A revisão sistemática de literatura possibilitou a este trabalho uma visão mais abrangente - um panorama geral - dos diferentes contextos e participantes que estão investigando a implementação dos exergames na educação, no intuito de desenvolver esta pesquisa que teve como objetivo geral mapear a produção sobre a utilização dos exergames na educação.

Por se tratar de uma tecnologia relativamente recente, a produção sobre a implementação dos exergames em âmbito educacional e suas implicações ainda é escassa, mas em crescente interesse acadêmico, tendo em vista as potencialidades dos exergames como ferramenta facilitadora da aprendizagem.

Como resultado das buscas, foram encontrados trabalhos desenvolvidos nos países Austrália, Canadá, China e Estados Unidos da América. Contudo, não foram encontrados trabalhos realizados em âmbito nacional, mas devido à popularização e à acessibilidade dos consoles de videogames caseiros, neste caso o Kinect para Xbox 360, as possibilidades de implementação deste objeto técnico em âmbito educacional têm aumentado, possibilitando uma prática diferenciada do cotidiano das aulas de Educação Física na escola.

Em sua grande maioria, os trabalhos encontrados foram desenvolvidos com a participação de crianças e adolescentes, onde apenas um trabalho foi realizado com universitários. Diante disso, vê-se a necessidade da investigação da implementação dos exergames com pessoas adultas, sejam elas de qualquer nível de ensino, para contribuição de novos achados sobre a temática, suprimindo essa lacuna deixada na literatura.

Os games utilizados foram todos comerciais, simulando ações cotidianas e fictícias com situações-problemas a serem resolvidas pelo jogador para consequente desenvolvimento da partida. A partir do realismo e da imersão proporcionada pelos exergames, aspectos importantes podem ser trabalhados no domínio da tomada de decisão a partir dos movimentos do corpo humano como mecanismo de interação com o game, atribuindo benefícios tanto físicos quanto cognitivos à prática do exergame.

Todos os trabalhos encontrados chegaram a um ponto em comum em que, através dos seus resultados, pode-se afirmar que a prática dos exergames possibilita o aumento do nível de atividade física, chegando a níveis moderados e vigorosos. A produção ainda é limitada e os resultados são preliminares. Diante disso, há a necessidade de que mais investigações sejam realizadas, envolvendo diferentes desenhos experimentais com maior duração das intervenções e com a participação de 
pessoas de diferentes níveis de ensino, para que possa haver a contribuição na replicação ou na refutação dos achados, a fim de consolidar os resultados já existentes na literatura.

A inclusão dos exergames como ferramenta potencializadora no processo de ensino e aprendizagem está condicionada à capacitação dos profissionais para a sua utilização adequada. Portanto, este estudo apresenta informações iniciais sobre a temática, a fim de tecer aproximações a serem desbravadas por aqueles que pretendem utilizar os exergames em âmbito educacional.

A maior contribuição desse trabalho é a própria revisão sistemática da literatura, que poderá servir como eixo norteador para futuros estudos, tendo como base os estudos primários elencados e analisados e os resultados apresentados na presente pesquisa. Como futuros trabalhos, pode-se direcionar para a implementação melhor sistematizada, com maior tempo de duração das intervenções e abrangendo diferentes níveis de ensino, a fim de consolidar os dados referentes às implicações relativas à implementação dos exergames em âmbito educacional, proporcionando mais uma ferramenta de apoio didático-pedagógico ao professor no processo de ensino-aprendizagem.

\section{REFERÊNCIAS}

ALVES, L. Game over: jogos eletrônicos e violência. São Paulo: Futura, 2005.

BARACHO, A; GRIPP, F; LIMA, M. Os exergames e a educação física escolar na cultura digital. Rev. Bras. Ciênc. Esporte, v. 34, n. 1, p. 111-126, 2012. Disponível em: <http://www.scielo.br/scielo.php?script=sci_arttext\&pid=S010132892012000100009>. Acesso em: 17 dez. 2015.

BARBOZA, E.; SILVA, A. A evolução tecnológica dos jogos eletrônicos: do videogame para o newsgame. In: $5^{\circ}$ Simpósio Internacional de Ciberjornalismo, 2014. Campo Grande. Anais... Campo Grande, 2014. Disponível em: <http://www.ciberjor.ufms.br/ciberjor5/files/2014/07/eduardo.pdf >. Acesso em: 14 fev. 2016.

CLEVENGER, K.; HOWE, C. Energy Cost and Enjoyment of Active Videogames in Children and Teens: Xbox 360 Kinect. Games for health: research, development, and clinical applications, v. 4, n. 4, p. 318-324, 2015. Disponível em: <http://online.liebertpub.com/doi/full/10.1089/g4h.2014.0101>. Acesso em: 17 jul. 2015. 
CORRÊA, A. et al. Realidade virtual e jogos eletrônicos: uma proposta para deficientes. In: MONTEIRO, C. Realidade virtual na paralisia cerebral. São Paulo: Plêiade, 2011.

FERES NETO, A. Videogame e educação física/ciências do esporte: uma abordagem à luz das teorias do virtual. In: Congresso internacional de ciências do esporte, 2.; Congresso brasileiro de ciências do esporte, 15., Recife, 2007. Anais... Goiânia: Colégio Brasileiro de Ciências do Esporte, 2007. Disponível em:

<http://www.cbce.org.br/docs/cd/resumos/062.pdf>. Acesso em: 9 dez. 2015.

FROSI, F; SCHLEMMER, E. Jogos digitais no contexto escolar: desafios e possibilidades para a prática docente. Anais do IX Simpósio Brasileiro de Jogos e Entretenimento Digital (SBGames), 2010. Disponível em: <http://www.sbgames.org/papers/sbgames10/culture/full/full13.pdf $>$. Acesso em: $3 \mathrm{dez}$. 2015.

GEE, J. Good video games and good learning: collected essays on video games, learning, and literacy. New York: Peter Lang, 2014.

GEE, J. What video games have to teach us about learning and literacy. New York: Palgrave Macmillan, 2007.

GULARTE, D. Jogos eletrônicos: 50 anos de interação e diversão. Rio de Janeiro: Novas Ideias, 2010.

HUIZINGA, J. Homo ludens: o jogo como elemento da cultura. 8 ed. São Paulo: Perspectiva, 2014.

LEMOS, A. Cibercultura: tecnologia e vida social na cultura contemporânea. Porto Alegre: Sulina, 2002.

LÉVY, P. Cibercultura. Rio de Janeiro: Editora 34, 1999.

LIN, J. "Just Dance": The effects of exergame feedback and controller use on physical activity and psychological outcomes. Games for health: research, development, and clinical applications, v. 4, n. 3, p. 183-189, 2015. Disponível em:

<http://online.liebertpub.com/doi/full/10.1089/g4h.2014.0092>. Acesso em: 17 jul. 2015.

MACARTHUR, B. et al. Active Videogaming Compared to Unstructured, Outdoor Play in Young Children: Percent Time in Moderate-to Vigorous-Intensity Physical Activity and Estimated Energy Expenditure. Games for health: research, development, and clinical applications, v. 3, n. 6, p. 388-394, 2014. Disponível em: < http://online.liebertpub.com/doi/full/10.1089/g4h.2014.0017>. Acesso em: 10 ago. 2015.

MELLECKER, R.; MCMANUS, A. Habituation and cardiovascular responses to the Gamercize stepper in Hong Kong Chinese girls. Games for health: research, development, and clinical applications, v. 2, n. 2, p. 70-74, 2013. Disponível em: <http://online.liebertpub.com/doi/full/10.1089/g4h.2012.0076>. Acesso em: 10 ago. 2015. 
MOITA, F. Game on: jogos eletrônicos na escola e na vida da geração @. São Paulo: Alínea, 2007.

PAPASTERGIOU, M. Exploring the potential of computer and video games for health and physical education: a literature review. Computers \& Education, v. 53, n. 3, p. 603-622, 2009. Disponível em:

<http://www.sciencedirect.com/science/article/pii/S0360131509000840>. Acesso em: 8 jan. 2016.

PAULA, B. Adaptando e desenvolvendo jogos para uso com o Microsoft

Kinect. Proceedings of SBGames, 2011. Disponível em:

$<$ http://www.sbgames.org/sbgames2011/proceedings/sbgames/papers/tut/1-

kinect_FAAST\%20_Final_MesmoComColunas.pdf $>$. Acesso em: 9 dez. 2015.

READING, S.; PRICKETT, K. Evaluation of children playing a new-generation motion-sensitive active videogame by accelerometry and indirect calorimetry. Games for health: research, development, and clinical applications, v. 2, n. 3, p. 166-173, 2013. Disponível em: <http://online.liebertpub.com/doi/full/10.1089/g4h.2013.0021>. Acesso em: 10 ago. 2015.

ROSENBERG, M. et al. New-generation active videogaming maintains energy expenditure in children across repeated bouts. Games for health: research, development, and clinical applications, v. 2, n. 5, p. 274-279, 2013. Disponível em: < http://online.liebertpub.com/doi/full/10.1089/g4h.2013.0037>. Acesso em: 17 jul. 2015.

SHAFER, D.; CARBONARA, C. Examining enjoyment of casual videogames. Games for health: research, development, and clinical applications, v. 4, n. 6, p. 452-459, 2015. Disponível em: <http://online.liebertpub.com/doi/full/10.1089/g4h.2015.0012>. Acesso em: 10 ago. 2015.

SOTHERN, M. Obesity prevention in children: physical activity and nutrition. Nutrition, v. 20, n. 7, p. 704-708, 2004. Disponível em: <http://www.nutritionjrnl.com/article/S0899-9007(04)00098-X/abstract>. Acesso em: 18 dez. 2015.

VAGHETTI, C.; BOTELHO, S. Ambientes virtuais de aprendizagem na educação física: uma revisão sobre a utilização de Exergames. Ciências e Cognição, v. 15, n. 1, p. 64-75, 2010. Disponível em:

<http://www.cienciasecognicao.org/revista/index.php/cec/article/view/292>. Acesso em: 5 jan. 2016.

WORLD HEALTH ORGANIZATION. Global Recommendations on Physical Activity for Health. Geneva, World Health Organization, 2010. Disponível em: <http://apps.who.int/iris/bitstream/10665/44399/1/9789241599979_eng.pdf>. Acesso em: 13 fev. 2016. 


\section{Como referenciar este artigo}

FERREIRA, Adilson Rocha.; FRANCISCO, Deise Juliana. Explorando o potencial dos jogos digitais: uma revisão sobre a utilização dos exergames na educação. Revista Ibero-Americana de Estudos em Educação, Araraquara, v. 12, n. esp. 2, p.1177-1193, ago./2017. Disponível em: <http://dx.doi.org/10.21723/riaee.v12.n.esp.2.10288>. EISSN: 1982-5587.

Submetido em: 30/04/2017

Aprovação final em: 28/06/2017 